\title{
Transitions Between Symmetric and Nonsymmetric Regimes in Binary-Mixture Convection
}

\author{
Esteban Meca $^{1}$, Isabel Mercader, Laureano Ramírez-Piscina \\ Departament de Física Aplicada, Universitat Politècnica de Catalunya, Jordi Girona 1-3, \\ E-08034 Barcelona, Spain
}

\begin{abstract}
We present here a comprehensive picture of the different bifurcations found for small to moderate Rayleigh number in binary-mixture convection with lateral heating and negative separation ratio $(S)$. The present work connects the symmetric regime found for pure fluid $(S=0)[1]$ with the fundamentally nonsymmetric regime found for $S=-1[2,3]$. We give a global context as well as an interpretation for the different associations of bifurcations found, and in particular we interpret an association of codimension-two bifurcations in terms of a higher codimension bifurcation never found, to our knowledge, in the study of an extended system.
\end{abstract}

Keywords: Double difussive convection, Binary mixture, Nonlinear dynamics, Dynamical systems, Codimension-three bifurcation

\section{Introduction}

The study of convecting systems has a long and rich history, and many of the techniques developed for their study have been later successfully applied to broader categories of problems, such of pattern-forming systems [4]. In

Email addresses: meca@wias-berlin.de (Esteban Meca), isabel@fa.upc.edu (Isabel Mercader), laure@fa.upc.edu (Laureano Ramírez-Piscina)

${ }^{1}$ Current address: Weierstrass Institute for Applied Analysis and Stochastics, Mohrenstr. 39, 10117 Berlin, Germany

Physica D 303 (2015) 3949, http://dx.doi.org/10.1016/j.physd.2015.03.009

(C) 2015, Elsevier. Licensed under the Creative Commons Attribution-NonCommercialNoDerivatives 4.0 International http://creativecommons.org/licenses/by-nc-nd/4.0/ 
particular, the study of the different instabilities has motivated some of the developments in the field of dynamical systems theory which in turn has led to a systematic way of approaching the study of convecting systems.

The study of convection in two-component systems, usually referred to as double-diffusive convection, was originally motivated by oceanographic problems [5], i.e. by the study of the relative role played by heat and solute (salt) diffusion in the development of convecting structures in the ocean. The application of double-diffusive convection to this and other problems, ranging from alloy solidification to stellar physics has received much attention in the past $[6,7,8]$.

In this paper we study a binary-mixture, that is, a fluid with two miscible components in which thermodiffusion (Soret effect) is present. The influence of the Soret effect as a driving force that could build up solutal gradients was investigated in [9], and the phenomenology uncovered and experimentally confirmed showed that the system behaved similarly to double-diffusive systems. This coincidence was later explained [10] by noticing a correspondence between Soret convection and the double-diffusive case for certain boundary conditions. This correspondence does not apply in this work.

While the most common setup in convection experiments is that of a fluid layer heated from below, other configurations are possible. In many situations (technological, oceanographical, etc.) lateral thermal gradients arise. The case of lateral thermal and solutal gradients is the one that concerns us in the present work. The behavior of a double-diffusive system heated from the side was also described by Stern [11] and Thorpe et al. [12].

With lateral heating and a laterally imposed concentration gradient, convection develops without threshold except in a particular limit in which solute buoyancy is exactly compensated with thermal buoyancy, thus making possible the existence of a purely diffusive solution. This case was studied in [13] and then in detail in [14] for low Rayleigh number, establishing the nature of the first bifurcation from this quiescent state. A subsequent work [15] studied the dependence of the first bifurcation on the aspect ratio and in another work [16] the bifurcation diagram of the steady solutions for low Rayleigh number was computed for different inclinations of the cavity, finally connecting with the horizontal problem.

The genericity of this particular case of compensating gradients was tested in [17]. It was proved that the bifurcation diagram is generic, except for the purely diffusive state which is destroyed when we move away from the compensating gradients case. This system has been further studied in [18], 
where the influence of the third dimension is studied for different aspect ratios. In $[19,20]$ the multiplicity of steady states in the large aspect ratio containers of Ref. [16] is studied and interpreted in terms of localized steady states (convectons).

In the case of lateral heating but without imposing a lateral concentration gradient a similar quiescent state can be obtained, in this case due to the solutal gradient built because of the Soret effect [2]. Note that this case is inequivalent to the previous one. Close to the threshold of the instability of the purely diffusive state, the behavior was found to be similar to the one found in the double-diffusive case. Nevertheless, as the Rayleigh number is increased, a richer variety of scenarios has been found. Most interestingly the dynamics is dominated by a low-frequency nonsymmetric periodic solution which appears in a global bifurcation, experiences multiple transformations and finally disappears in a blue sky catastrophe [2].

This behavior is in sharp contrast with the one found in the pure fluid case, for the same values of the Prandtl number [21, 1]. In that case, for the same range of explored Rayleigh numbers, the dynamics remains symmetric and steady, and loses stability in a Hopf bifurcation to a short-period symmetric solution, which disappears for much higher values of the Rayleigh number in a very complex scenario leading to a chaotic attractor [1].

To explore the genericity of the results of [2] and to bridge the gap with the pure fluid case, a study of the successive transformations of the global connection that gives birth to the aforementioned low-frequency nonsymmetric periodic solution was undertaken in [3]. It was found that this global connection changed in nature from a saddle-loop to a SNIC and then again to a saddle-loop, finally to become a Hopf bifurcation in a Takens-Bogdanov (TB) point.

This Takens-Bogdanov point was found to be associated with a saddlenode-separatrix loop (SNSL) bifurcation and with a cusp. This conjunction of codimension-two bifurcations has been found in many problems of very different nature, from systems biology [22] to lasers [23]. In [3] it was found that this conjunction and all the low-Rayleigh number dynamics could be described as a part of the unfolding of a degenerate (codimension-three) Takens-Bogdanov point, the saddle case found by Dumortier et al. [24].

In this paper we present a comprehensive picture of the transformations that the bifurcation diagram undergoes as we move away from the case of compensating gradients to the case of pure fluid. To this end a wide range of variation of parameters (Rayleigh number and separation ratio) is ex- 
plored, and different numerical techniques (mainly temporal integration of dynamical equations and continuation of steady solutions) are employed. In particular we pay a special attention to the disappearance of the long-period nonsymmetric solution and to the change of character of the bifurcations from global to local. This kind of investigation is hampered by the long temporal scales of the associated dynamics and the complexity of the different scenarios found, and indeed an arduous numerical effort has been necessary to complete the task. Moreover, the range of parameters spanned is quite large and some of the most interesting and complex dynamics occurs in very small regions of parameter space. Luckily, as in Ref. [3], the topology of the bifurcation lines could be rationalized as associations of codimension-two bifurcations that could be linked to higher codimension phenomena, which in fact resulted to be a guide to find and organize them. We think that such connections constitute an important result of this study.

The structure of the paper is as follows. In Sec. 2 we state the system under study, its equations and the numerical methods employed. In Sec. 3 we present the results for the different ranges of parameters explored, and discuss the structure of the bifurcation diagrams. Finally we present the conclusions in Sec. 4.

\section{Formulation of the problem}

Binary mixtures are characterized by a cross-diffusion effect called the Soret effect that describes the diffusive separation of the lighter and heavier components of the mixture in an imposed temperature gradient. Specifically, if $c$ is the concentration of the heavier component, its flux is proportional to $-\left(\nabla c+c(1-c) S_{T} \nabla T\right)$, where $S_{T}$ is the Soret coefficient, and $T$ is the temperature; then, when $S_{T}$ is negative the heavier component migrates, on a diffusive time scale, towards the hotter boundary.

In our work, we consider a binary mixture in a 2-D rectangular cavity of aspect ratio $\Gamma=d / h=2$, where $d$ is the length and $h$ is the height of the cavity. A difference of temperature $\Delta T$ is maintained between the right and left vertical walls $\left(T_{r}-T_{l}=\Delta T\right)$ and at the horizontal walls, which are assumed to be perfectly conducting, a linear temperature profile between the two prescribed temperatures is imposed. All walls are considered no-slip and impervious (no mass flux) boundaries.

The Boussinesq equations describing the system are nondimensionalized using $\Delta T$ as unit of temperature, $h$ as the unit of length and the thermal 
diffusion time in the vertical direction $t_{\kappa}=h^{2} / \kappa$ as the unit of time, $\kappa$ being the thermal diffusivity. In the equation of mass-conservation we approximate the expression of the flux of the heavier component substituting the concentration $c$ appearing in the thermodiffusion term by its concentration $c_{0}$ in the homogeneous mixture and thus the concentration field is scaled by $\Delta c=-c_{0}\left(1-c_{0}\right) S_{T} \Delta T$.

With the stated approximations the dimensionless equations explicitly read

$$
\begin{array}{rll}
\partial_{t} \mathbf{u}+(\mathbf{u} \cdot \nabla) \mathbf{u} & = & -\nabla P+\sigma \nabla^{2} \mathbf{u} \\
+\sigma R a[ & (1+S) & (-0.5+x / \Gamma)+\theta+S C] \hat{\mathbf{z}}, \\
\partial_{t} \theta+(\mathbf{u} \cdot \nabla) \theta & = & -v_{x} / \Gamma+\nabla^{2} \theta \\
\partial_{t} C+(\mathbf{u} \cdot \nabla) C & = & -v_{x} / \Gamma-\tau \nabla^{2}(\theta-C), \\
\nabla \cdot \mathbf{u} & =0,
\end{array}
$$

where $\mathbf{u} \equiv\left(v_{x}, v_{z}\right)$ is the velocity field in $(x, z)$ coordinates, $P$ is the pressure over the density, and $\theta$ denotes the dimensionless departure of the temperature from a linear horizontal profile. $C$ is the scaled deviation of the concentration of the heavier component relative to the linear horizontal profile which would develop in a pure diffusive state. The dimensionless parameters are the Prandtl number $\sigma=\nu / \kappa$, the Rayleigh number $R a=\alpha g h^{3} \Delta T / \nu \kappa$, the Lewis number $\tau=D / \kappa$, where $\nu$ denotes the kinematic viscosity, $g$ the acceleration of gravity, $\alpha$ the thermal expansion coefficient, and $D$ is the mass diffusivity. The separation ratio $S$ is defined as $S=c_{0}\left(1-c_{0}\right) \frac{\beta}{\alpha} S_{T}$ where $\beta$ is the mass expansion coefficient ( $\beta>0$ for the heavier component).

Boundary conditions are written as

$$
\mathbf{u}=\theta=\mathbf{n} \cdot \nabla(\theta-C)=0, \quad \text { at } \partial \Omega
$$

Note that there does not exist a redefinition of the pressure that would eliminate the terms that depend on $x$ in Eq. (1). This means that the conductive solution given by the linear profiles of concentration and temperature $(\mathbf{u}=\theta=C=0)$ typically does not exist. The lateral inhomogeneity in the density will always lead to buoyancy forces that cannot, in general, be compensated by changes in the pressure of the surrounding liquid. There is only one exception to this behavior, and is the case in which $S=-1$. In this case there exists a purely conductive solution. 
Eqs.(1-4), together with boundary conditions (5), remain invariant under the transformation $\pi$, a central symmetry around the point $(\Gamma / 2,1 / 2)$,

$$
\begin{aligned}
\pi:\left(v_{x}, v_{z}, \theta, C\right) & \rightarrow\left(-v_{x},-v_{z},-\theta,-C\right), \\
(x, z) & \rightarrow(\Gamma-x, 1-z) .
\end{aligned}
$$

This is a generalized reflection and the group $\{\pi, I\}$ is isomorphic to $\mathbb{Z}_{2}$. Hence any solution of these equations either is $\pi$-invariant (which from now will be called symmetric) or its image under $\pi$ is also a solution (constituting a pair of nonsymmetric solutions). This has important consequences on the nature of its possible bifurcations [25].

Eqs. (1-4) and boundary conditions (5) have been solved by a second order time-splitting algorithm, proposed by Hugues and Randriamampianina [26], applied to a pseudo-spectral Chebyshev method. To calculate steady solutions, we have adapted a pseudo-spectral first-order time-stepping formulation to carry out Newton's method [27, 28, 29, 30]. In the preconditioned version of Newton's iteration, the corresponding linear system is solved by an iterative technique using a GMRES package [31]. The linear stability analysis of the steady states is conducted by computing the leading eigenvalues of the Jacobian by means of Arnoldi's method, using routines from the ARPACK package.

For numerical calculations the chosen parameters have been Prandtl number $\sigma=0.00715$ and Lewis number $\tau=0.03$, close to those characteristic of molten gallium-doped germanium [32, 33]. The system has been discretized in space by using $60 \times 30$ and $72 \times 48$ mesh points for values of the Rayleigh number of order $R a \approx 1000$, and $90 \times 60$ and $120 \times 60$ mesh points for $R a \approx 2000$. The accuracy of the results was estimated by evaluating the change of the Rayleigh number at all the bifurcation points when changing the numerical parameters within the mentioned ranges, both for those calculated with the Arnoldi Method (steady-state bifurcations) and for those obtained with the time dependent code, and in the worst case it was better than $0.1 \%$.

\section{Results}

In this section we present the results obtained for large variations of the separation ratio $S$, spanning values from -1 to 0 , and Rayleigh number $R a$, from 0 to 2500. The complete (except for very small features not visible at 


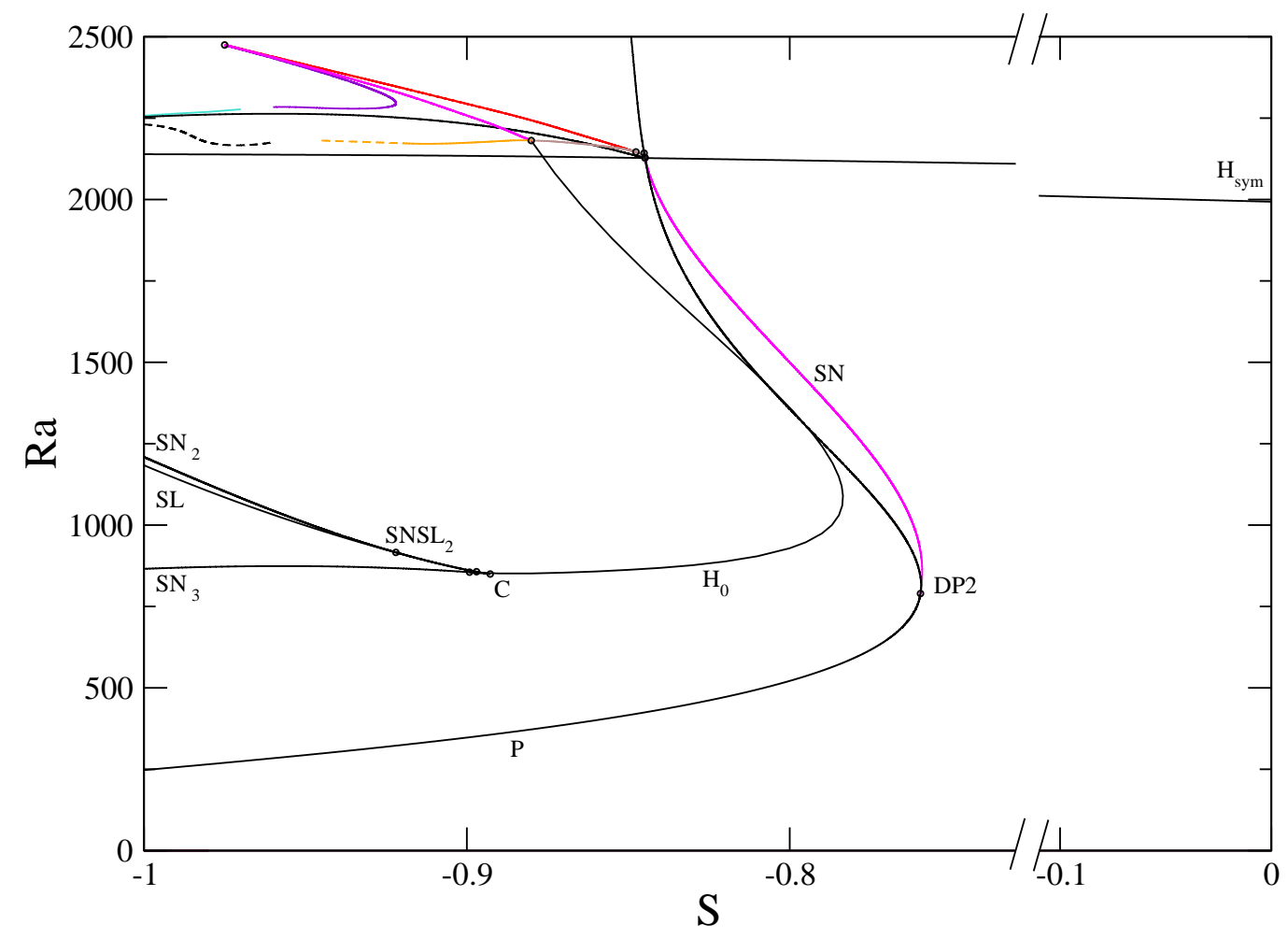

Figure 1: Complete bifurcation diagram for $-1 \leq S \leq 0$. We present below enlargements for the most relevant regions. In particular, see Figs. 9 and 10 for additional detail in the upper $R a$ region.

this scale) bifurcation diagram can be seen in Fig. 1. We have structured the description of the results by focusing on different regions of this diagram. In Subsecs. 3.1 and 3.2 we review previously published results [2, 3] respectively for $S=-1$ and for low $R a$ and $S$ near -1 . The solutions and bifurcations found in those regions are the starting point for studying the transformations of the dynamics to the very simple scenario existing for the pure fluid at $S=$ 0. In Subsec. 3.3 we describe the low $R a$ regime, in which the nonsymmetrical solutions disappear by increasing $S$. In Subsecs. 3.4 and 3.5 we explore the complex scenario that occurs for high $R a(R a=2000$ to $R a=2500)$. In this regime, increasing $S$ makes the bifurcation diagram simpler until only a Hopf bifurcation of symmetric solutions is found at $S=0$. 


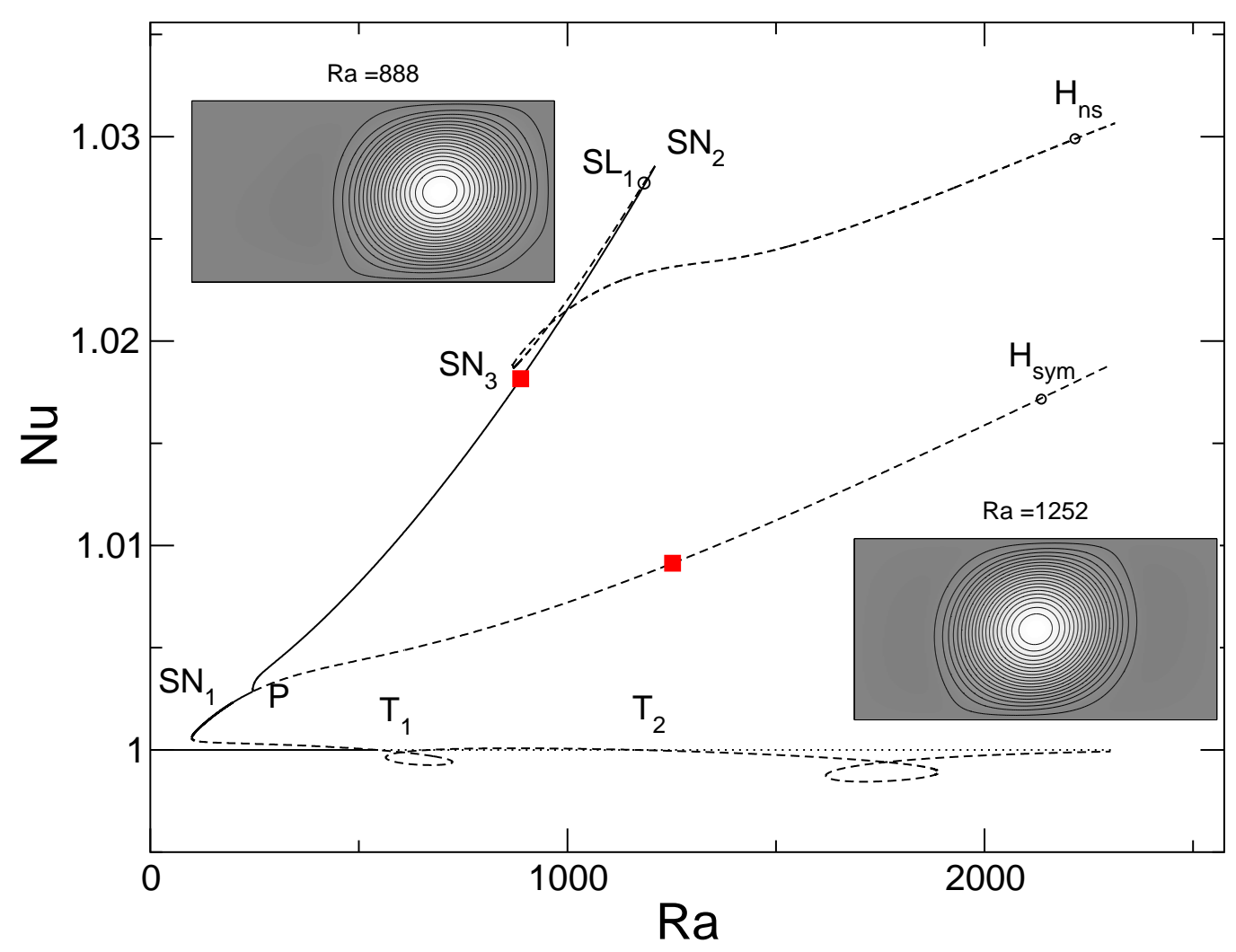

Figure 2: Nusselt number (evaluated on the left side) as a function of $R a$ for the computed steady solutions at $S=-1$. Stable (unstable) solutions are represented with solid (dashed) lines. The nonsymmetric branches near $T_{1}$ and $T_{2}$ are not visible at this scale. In the two insets, streamfunction patterns corresponding to the points marked with red squares are shown, one in the symmetric and another in the nonsymmetric branch.

\subsection{Results for $S=-1$}

In this subsection we review previous results obtained for $S=-1$. As it has already been mentioned, this precise value of the separation ratio allows for a quiescent, purely conductive solution, but as the $R a$ number increases the system exhibits a rich and interesting behavior. In Fig. 2 we show a summary of the steady solutions found in the system and their respective bifurcations. We characterize each steady solution for each value of $R a$ by its Nusselt number, defined as the quotient of heat flux through the left side of the container divided by the heat flux of the corresponding quiescent solution.

For small $R a$, the aforementioned conductive solution is found (line $N u=$ 


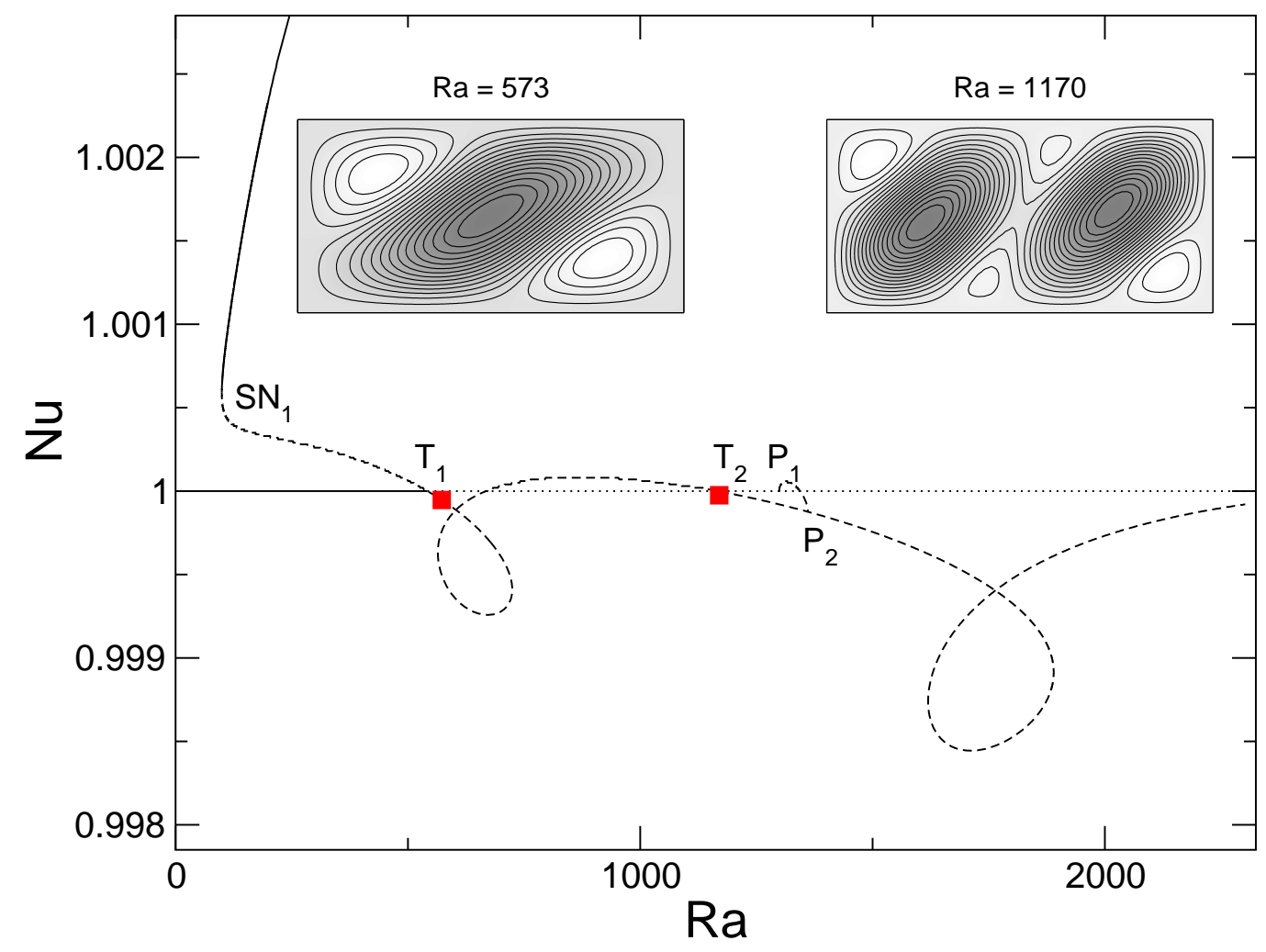

Figure 3: Nusselt number (evaluated on the left side) as a function of $R a$ for the lower (supercritical) branch of symmetric solutions at $S=-1$. In the inset, streamfunction patterns corresponding to the points marked with red squares are shown. Note that another nonsymmetric branch appears near $T_{1}$ much like the one near $T_{2}$, but it is not visible at this scale. 
1 in Fig. 2), which is stable until a symmetry-preserving transcritical bifurcation $T_{1}$ occurs at $R a=541.9$. This area of the bifurcation diagram is shown in more detail in Fig. 3. The supercritical branch is characterized by a very weak convection (resulting in Nusselt numbers very close to 1), which in the beginning forms a pattern with a main clockwise roll accompanied by a pair of counter-clockwise smaller rolls (see inset in Fig. 3). This branch becomes unstable in a subcritical pitchfork bifurcation (not shown in the figure) for $R a=542.4$. The nonsymmetric branch born in the pitchfork bifurcation ends in another pitchfork bifurcation of the conductive state also very close to $T_{1}$. This is a scenario very similar to that of double diffusive convection with $N=-1$.[17]. The supercritical branch, after losing stability, undergoes two additional saddle-node bifurcations and ends up meeting the conductive solution again at another transcritical bifurcation point, $T_{2}$, as shown in Fig. 3. There we find a similar structure of bifurcations, with a nonsymmetric solution connecting again another pair of pitchfork bifurcations. In the process the convective pattern gains a roll (see inset). This is repeated successively for larger $R a$.

The most interesting dynamics originates from the strongly subcritical branch born at $T_{1}$. This branch gains stability at a turning point $\left(S N_{1}\right)$ with a very small Rayleigh number $\left(R a_{S N_{1}}=99\right)$ and loses it again in a pitchfork bifurcation $(P)$ at $R a_{P}=245$ (see Fig. 2). At $P$ a nonsymmetric solution is born, which after the occurrence of $T_{1}$ is the only remaining stable steady solution present in the system. In the insets in Fig. 2 representative streamfunction patterns of both branches are shown. Both show strong convection, with the symmetric solution characterized by a single, counter-clockwise centered roll. The nonsymmetric solution has a single roll, very similar to the symmetric one, but displaced to one side. This nonsymmetric solution loses stability in a saddle node bifurcation $S N_{2}$ for $R a_{S N_{2}}=1209$, and becomes more unstable in another saddle node bifurcation $S N_{3}$ for $R a_{S N_{3}}=865.6$ (see Fig. 2). This branch becomes further unstable still in a Hopf bifurcation $\left(H_{n s}\right)$ at $R a_{H_{n s}}=2218$ with a frequency $\omega_{H_{n s}}=6.80$. Similarly, the symmetric branch undergoes a Hopf bifurcation $\left(H_{s y m}\right)$ at $R a_{H_{s y m}}=2137$ with a frequency $\omega_{H_{s y m}}=6.73$. Both bifurcations have a very similar frequency, giving a hint of a possible link between the two bifurcations as the parameters are varied (see Sec. 3.5).

For $R a>R a_{S N_{2}}$ no more stable steady solutions are found in the system. Beyond this value of $R a$, the time dependent evolution code reveals the presence of a periodic stable solution for a wide range of $R a$, connected 


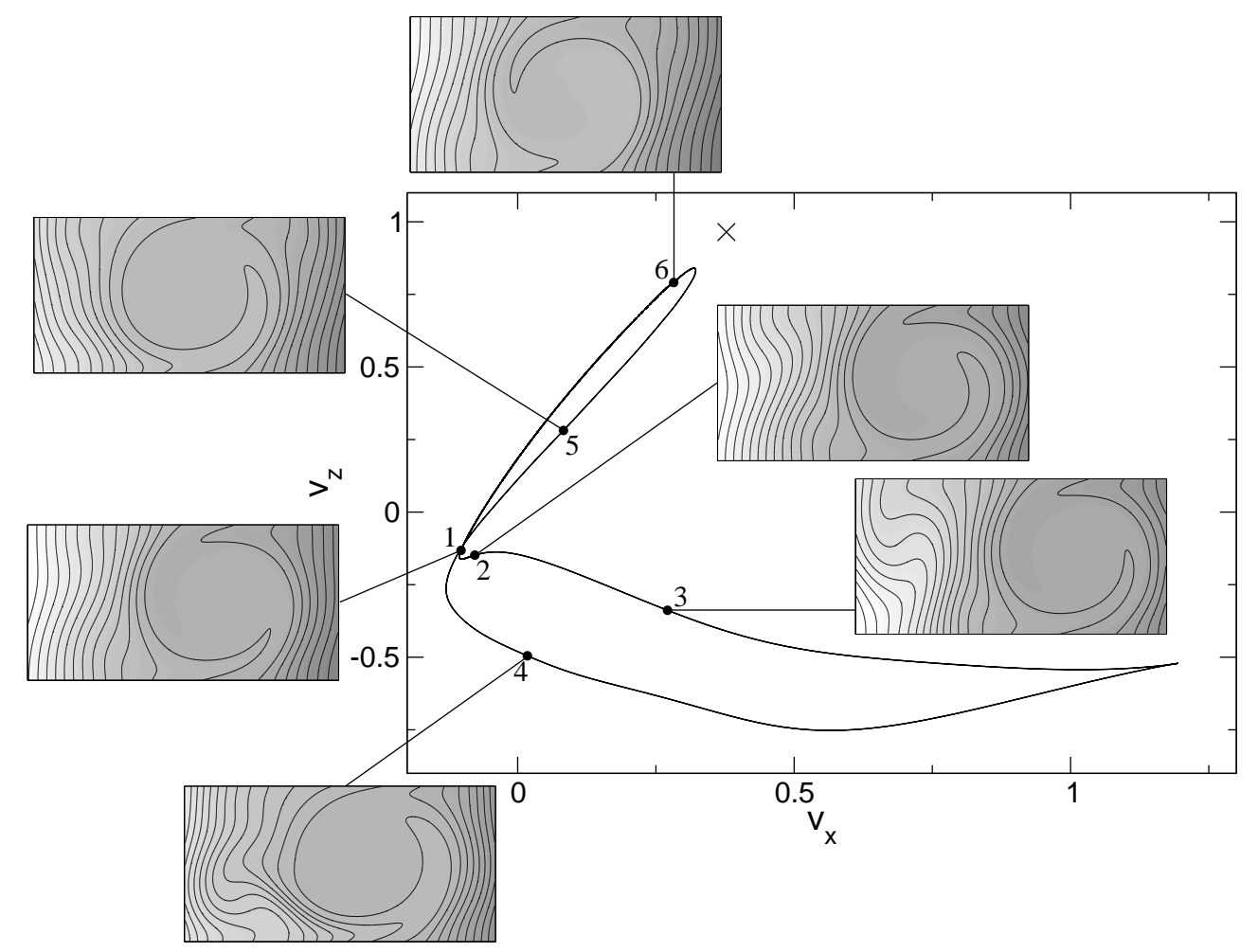

Figure 4: Component $z$ of the velocity vs. component $x$ at a representative point for $R a=1900$ for the LPNS orbit at $S=-1$. The corresponding concentration patterns at different points of the orbit are also shown. The location of the steady symmetric unstable solution is shown as a cross. Times of the snapshots: $t_{1}=0.0, t_{2}=8.0, t_{3}=12.0$, $t_{4}=18.0, t_{5}=22.0$, and $t_{6}=30.0$, for a period of $T=380.61$. 
with the nonsymmetric steady branch through a global bifurcation, namely a homoclinic saddle-loop connection at $R a_{S L_{1}}=1183.67$, indicated by $S L_{1}$ in Fig. 2. The nature of the bifurcation, i.e. the fact that it is a saddle-loop, can be confirmed by the logarithmic law that the period follows when approaching the critical point. The evolution of the velocity at a representative point and the changes in the concentration field along this long-period nonsymmetric (LPNS) orbit are represented in Fig. 4. When the system is near the unstable steady symmetric solution (represented by a cross in Fig. 4) the system is also nearly symmetric, with the large roll in the center, and with a slow dynamics during which the roll is displacing towards one side forming larger solute gradients, reaching a state very similar to the nonsymmetric steady solution (not shown in Fig. 4). At a certain point the solute layer collapses, giving rise to a fast large excursion through phase space during which the solute redistributes and the roll returns to the centered position. This fast evolution shows itself as a large spike in the system response.

By increasing $R a$ the dynamics of the system becomes more complex. At first the LPNS orbit remains qualitatively similar until the unstable steady symmetric solution undergoes a Hopf bifurcation at $R a_{H_{s y m}}=2137$. While the symmetric orbit thus generated is unstable, this bifurcation changes the geometry of phase space and therefore influences the LPNS orbit. At this value of $R a$ the LPNS orbit starts to curl, presenting ripples of the same frequency than that of the unstable symmetric orbit. As the Rayleigh number increases, the LPNS orbit finally becomes unstable in a period-doubling bifurcation around $R a=2220$, and becomes rapidly chaotic in a period-doubling cascade. At this point, the period of the slow oscillations (well reproducible, with changes smaller than $0.01 \%$ ) increases dramatically with the Rayleigh number, making the ratio of the periods of the slow and the fast oscillations as large as 6000 for $R a=2255$. At this value of $R a$ the symmetric periodic orbit has become stable in a pitchfork bifurcation $(P P O)$ at $R a_{P P O}=2253$, and coexists with the stable attractor (see Fig. 5a). The long period of the attractor finally diverges following a square root law at $R a_{B S C}=2257.73$, where it disappears in a Blue Sky Catastrophe (BSC) bifurcation. The type of divergence is indicative of a collision with a saddle-node orbit. The simplest scenario accounting for such collision is depicted in Fig. 5b. In this scenario, the symmetric orbit that appears after $H_{\text {sym }}$ undergoes a pitchfork bifurcation (PPO). At this pitchfork bifurcation an unstable branch of nonsymmetric periodic orbits appears. Then, this orbit undergoes a saddle-node bifurcation $\left(\mathrm{SNO}_{2}\right)$ that makes it stable but shortly after it undergoes an- 

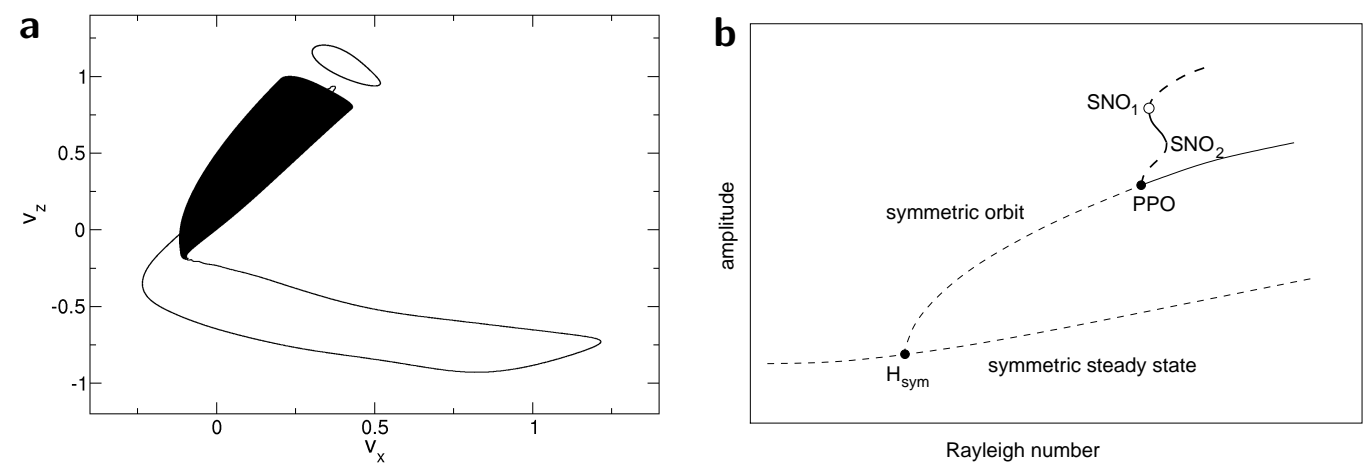

Figure 5: (a) Attractors at $R a=2255$ and $S=-1$. Two stable solutions are shown: the symmetric periodic orbit and the large (chaotic) attractor. (b) Schematic diagram of the successive bifurcations leading to the formation of a saddle-node of periodic orbits. The point where the connection with the BSC takes place is marked with an open circle.

other saddle-node bifurcation $\left(S N O_{1}\right)$. This saddle-node bifurcation would be located at $R a_{B S C}=2257.73$, at the point where the period diverges. One could expect that after the BSC the orbit would settle on the stable branch of $S N O_{1}$ for higher values of $R a$, but instead the orbit disappears into the symmetric stable orbit. This would be due to a very small separation between $S N O_{1}$ and $S N O_{2}$ or simply because of a very small basin of attraction of that orbit. Consistent with that collision is the fact that the (fast) winding frequency of the attractor and the frequency of the symmetric orbit right after $P P O$ are very similar, with a common value $\omega=7.01$.

\subsection{Results for low Rayleigh number and low $S>-1$}

The scenario sketched for $S=-1$ is not specific for this precise value of $S$ but nonetheless should undergo changes when varying this parameter in order to accommodate the simpler scenario found for a pure fluid [1], i.e. for $S=0$. In this subsection we review the transformations of the steady nonsymmetric branch, and in particular the eventual disappearance of the global bifurcation $S L_{1}$ that originates the LPNS orbit, together with the saddle nodes $S N_{2}$ and $S N_{3}$, until only a Hopf bifurcation remains. These results were discussed in detail in a previous work ([3]).

As we have seen above (see Fig. 2), for $S=-1$ there is a range of values of $R a$ for which the only stable solutions found are the steady nonsymmetric solution, whose stability ends at $S N_{2}$, and the LPNS orbit, connected to the former at the global bifurcation $S L_{1}$. At this collision the period of the orbit 
a

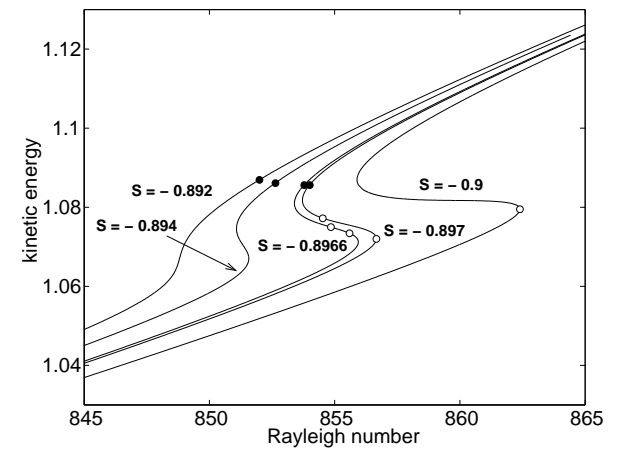

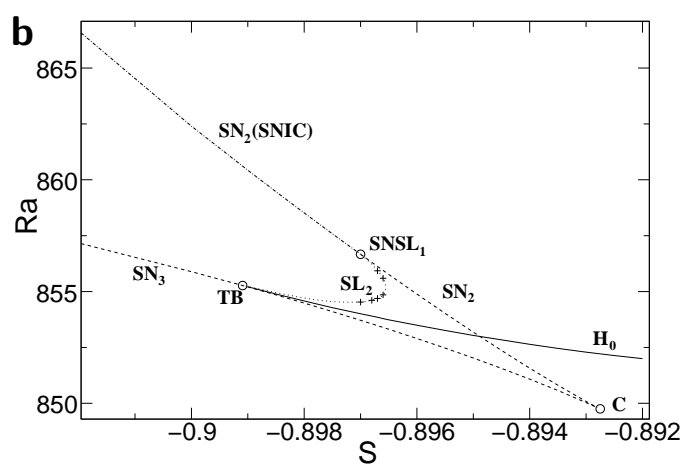

Figure 6: (a) Kinetic energy versus Rayleigh number for the steady nonsymmetric branch, for several values of $S$. Hopf bifurcations are shown as filled circles and global bifurcations (either $S L$ or $S N I C$ ) are shown as open circles. (b) Detail of the bifurcation diagram in the parameter space $(R a, S)$ near the cusp C. After [3].

presents a logarithmic divergence, thus confirming the saddle-loop nature of the connection. This part of the branch of steady solutions presents two turning points: $S N_{2}$, where the solution become unstable, and $S N_{3}$, where an additional eigenvalue becomes positive thus increasing its instability.

The transformations of these bifurcations when increasing $S$ can be analyzed following the results depicted in Fig. 6. The first qualitative change when $S$ increases occurs when the locus of the global connection $S L_{1}$ moves towards $S N_{2}$, until it meets the saddle-node at about $S=-0.922$, becoming a SNIC (saddle node on an invariant circle) global bifurcation. This is the situation depicted for $S=-0.9$ in Fig. 6a. In this case the connection point is also located by the divergence of the period, but the law ceases to be logarithmic to become of square-root type.

For larger $S$ new bifurcations of codimension-one appear. In Fig. 6a it is shown that for $S=-0.897$ two new bifurcations have appeared very close to the turning point $S N_{3}$, a Hopf bifurcation $\left(H_{0}\right)$ at which a periodic solution is born and, for a slightly higher value of $R a$, another saddle-loop bifurcation $\left(S L_{2}\right)$ at which the orbit born at $H_{0}$ collides with a saddle point. The simultaneous appearance of these two bifurcations is caused by a TakensBogdanov bifurcation (see below) between $S=-0.9$ and $S=-0.897$. The nonsymmetric steady state between $S N_{3}$ and $H_{0}$ has also become stable due to the same cause. If $R a$ is further increased, the nonsymmetric periodic orbit reappears in a $S N I C$ bifurcation at $S N_{2}$. 
When the value of $S$ is raised a little, up to $S=-0.8966$, the latter global $S N I C$ connection point detaches from $S N_{2}$, thus becoming a saddleloop again. At this moment we have a solution branch with two saddleloop connections, very close to each other. As before, this was tested by performing a fit to the logarithmic law for the divergence of the periods of the two connections.

If we turn now to Fig. 6a, we see that for $S=-0.894$, both global connections have disappeared and hence the orbit is created at $H_{0}$. If $S$ is further increased, $S N_{2}$ and $S N_{3}$ annihilate each other in a codimension-two cusp bifurcation that occurs at $S=-0.8928$. The final situation, in which only the Hopf bifurcation (with very low frequency) is found, is represented by the $S=-0.892$ branch of Fig. 6 a.

All of the previous results can be understood in terms of the different connections between codimension-two bifurcations in the $R a-S$ plane, as shown in Fig. 6b. The point near $S=-0.922$ at which the global connection attaches to the $S N_{2}$ point (not shown in Fig. 6b, see Fig. 1) is in fact a saddlenode separatrix-loop codimension-two bifurcation. We denote it by $S N S L_{2}$. This point is then the merging of the two bifurcation lines corresponding to the $S N_{2}$ and $S L_{1}$ bifurcations from $S=-1$, forming a $S N I C$ line until the global connection detaches from $S N_{2}$ near $S=-0.8970$. This detachment point is another $S N S L, S N S L_{1}$, and a line of homoclinic saddle-loop connections $\left(S L_{2}\right)$ is also born there. This line of global connections turns towards lower values of $S$, thus explaining the previous duplicity of saddleloop connections. The $S L_{2}$ line ends meeting the $S N_{3}$ line at the birth of the $H_{0}$ line at $S=-0.8990$ in a Takens-Bogdanov $(T B)$ point, which is characterized by the presence of a double zero eigenvalue. This codimension-two point explains the linked appearance of the $H_{0}$ and the second saddle-loop in figure 6 a for $S=-0.897$.

As $S$ is increased, the $S N_{3}$ and the $S N_{2}$ lines approach each other, until they finally meet at a cusp bifurcation point around $S=-0.8928$ (this bifurcation is denoted as $C$ ). For larger values of $S$, as it has previously been stated, the boundary of existence of the LPNS orbit is the $H_{0}$ line. Thus, the birth of this orbit has switched from a global to a local bifurcation, but for large enough values of $R a$ the origin of the orbit is indistinguishable, as the positive eigenvalues of the steady state become also complex conjugate for instance in $S=-1$.

The bifurcation diagram described above for this low-Rayleigh regime is remarkable in that it is not specific of this problem, but rather has a generic 
character[3]. Indeed it can be found as a slice in parameter space in the unfolding of a codimension-three degenerate Takens-Bogdanov point ([24]) also known as the Dumortier-Roussarie-Sotomayor $(D R S)$ bifurcation.

\subsection{Disappearance of the nonsymmetric regime}

In this subsection we will describe in detail how the nonsymmetric regime disappears when $S$ is increased further in the low $R a$ region. This low $R a$ region is shown in Fig. 7 . We are now in the right part of the complete diagram ( $R a-S$ diagram, Fig. 1 ), at values of separation ratio $S>-0.83$. For the smaller values of $R a$ we still have the line $P$ of pitchfork bifurcations of the symmetric solutions that gives rise to steady nonsymmetric solutions. Above this line we find the branch of Hopf bifurcations $H_{0}$ of these nonsymmetric solutions, which is the result of the transformations described in the previous subsection. The dynamics in this region of the bifurcation diagram is simpler than that of other regions discussed above, since the dynamics is controlled by only three branches of bifurcations (the already discussed $P$ and $H_{0}$ lines plus a new line of saddle node bifurcations $S N$ ) and one codimension-two point, $D P 2$. The Hopf bifurcations undergone by the nonsymmetric steady solution are of small frequency (see subsection 3.2). The $S N$ branch corresponds to the saddle-node points of the branches of steady nonsymmetric solutions, and $D P 2$ is a degenerate pitchfork located in the low $R a$ part of the pitchfork branch.

In the inset of Fig. 7 we show schematically the solutions and their stability as a function of the Rayleigh number for $S=-0.81$ (which is indicated by the vertical line in the $S$-Ra diagram). We can observe a branch of steady symmetric solutions that are unstable in the region between the two pitchfork bifurcations. These solutions lose stability again at $R a \approx 2200$ where they undergo Hopf bifurcations (not shown in this figure, they correspond to the curve $H_{\text {sym }}$ shown in Fig. 1). Steady nonsymmetric solutions exist from the low- $R a P$ (which is a supercritical pitchfork) to $S N$, and are unstable in the region delimited by the curve $H_{0}$. In this region stable periodic nonsymmetric solutions exist (the LPNS orbit). Between the high- $R a P$ (subcritical pitchfork) and SN there exist two different nonsymmetric steady solutions, one stable and the other unstable. This scenario persists until the turning point of the curve $H_{0}$, which occurs at $S \approx-0.783$. For less negative values of the separation ratio $S$, and for values of the Rayleigh number smaller than 2500 , there are no more periodic nonsymmetric orbits. 


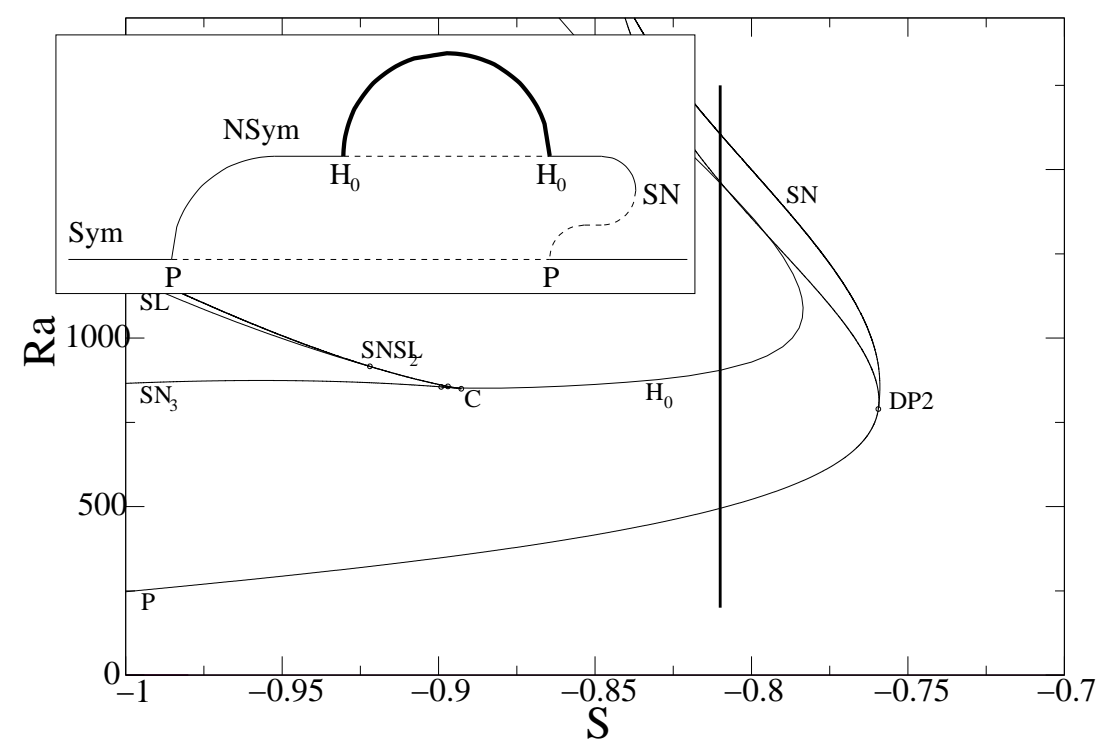

Figure 7: Bifurcation diagram for small $R a$. Vertical bar indicates the constant- $S$ cut in $(S-R a)$ space for which we present the diagram in the inset. Inset: schematic diagram of solutions and bifurcations found in the system for $S=-0.81$ as a function of $R a$.
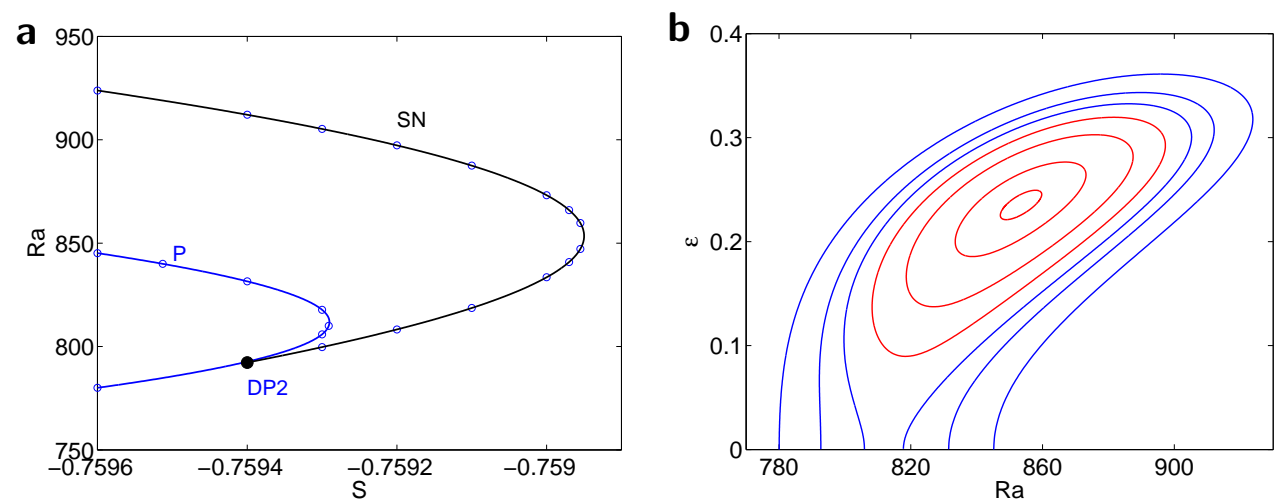

Figure 8: (a) Detail of the bifurcation diagram in the $(S, R a)$ parameter space near the degenerate pitchfork $D P 2$. $P$ stands for pitchfork bifurcations of the steady symmetric solutions, and $S N$ for saddle-node of the nonsymmetric steady solutions. (b) $\epsilon$ (parameter that indicates the symmetry breaking of nonsymmetric steady solutions) versus the Rayleigh number for different values of $S$. From outside to inside $S=$ $-0.7596,-0.7594,-0.7593,-0.7592,-0.7591,-0.7590,-0.758955$. Notice the isolas for the last four values. 
From $S=-0.783$ to $S=-0.7594$ the scenario is similar to that depicted in Fig. 7, except that now the two Hopf bifurcations and the branch of nonsymmetric periodic solutions are no longer present. At $S=-0.7594$ and $R a=792$, a codimension-two bifurcation takes place: a degenerate pitchfork (DP2), where the pitchfork of the lower branch changes from supercritical to subcritical (see this region in more detail in Fig. 8a). From that point on and for a fixed value of $S$, the region of values of Rayleigh number for which steady nonsymmetric solutions exist is limited by two saddle-node points. Namely, we find nonsymmetric stable solutions in all the $R a$ range between the two values of the $S N$ curve, and nonsymmetric unstable between each $S N$ and its closest point on the $P$ curve. At $S \approx-0.7593$ (turning point of curve $P$ ) the two subcritical pitchforks annihilate each other, and increasing further $S$ the branches of nonsymmetric solutions constitute isolas. These isolas collapse at $S \approx-0.75895$, which corresponds to the turning point of the $S N$ curve. This scenario is shown in Fig. $8 \mathrm{~b}$, where we have represented a parameter $\epsilon$, quantifying the symmetry breaking of a solution, as a function of the Rayleigh number for different values of $S$. The parameter $\epsilon$ is built as follows

$$
\epsilon=v_{z}\left(x_{o}, z_{o}\right)+v_{z}\left(\Gamma-x_{0}, 1-z_{0}\right)
$$

and hence the steady symmetric solutions lie on the horizontal line $\epsilon=0$. The intersection points of the curves with the $\epsilon=0$ axis are the pitchfork bifurcations from which the nonsymmetric solutions (which are represented here as continuous lines regardless of their stability) are born. The outer curve is for $S=-0.7596$ and the inner is for $S=-0.758955$.

\subsection{High Ra. Connection with $S=-1$}

If $S$ is increased in the high $R a$ regime, the picture presented in section 3.1 remains valid in a range of values of $S$ close to -1 . In Fig 9 we see a diagram of the bifurcations for that region in parameter space.

Again, the LPNS orbit loses stability in a period doubling or flip $(f)$ bifurcation, and very quickly becomes a chaotic attractor in a period doubling cascade as $R a$ is increased. Again, the divergence of the (average) long period of the attractor follows an inverse square-root law, which allows us to identify its disappearance as a Blue-Sky Catastrophe.

As $S$ is increased, the flip bifurcation moves slightly towards smaller values of $R a$, and for a high enough value of $S$ the LPNS orbit loses stability to a quasi-periodic solution, i.e. becomes a torus in a Neimark-Sacker bifurcation $\left(N S_{1}\right)$. The nature of this transition, marked in Fig. 9 by $B$, is not 


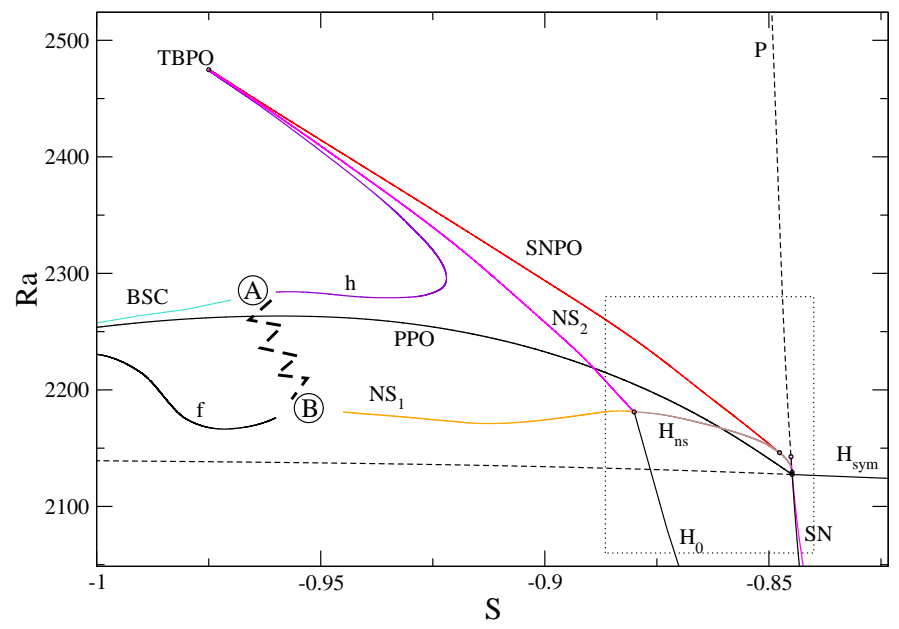

Figure 9: Enlargement of the relevant high Ra region of Fig. 1. Dashed lines correspond to bifurcations between unstable states, found by continuation. An enlargement of the region bounded by the dotted rectangle is presented in Fig. 10.

clear, for several reasons. First, this transition point is very hard to locate. We have located points where the long-period orbit becomes a torus, then for a slightly higher value of $R a$ it enters in a resonance region, and there it undergoes a period-doubling cascade, thus becoming a chaotic attractor. Also, the orbit can become chaotic following an initial flip bifurcation and a subsequent period-doubling cascade, only to follow a reverse period-doubling cascade for higher $R a$ and then become a torus in a $N S$ bifurcation. The regions where this mixed behavior occurs become narrower and narrower around point $B$, and hence it is hard to pinpoint a single codimension-two bifurcation as the reason for this behavior.

Also, the transition from the chaotic attractor to a torus (marked by a thick discontinuous broken line in Fig. 9) is problematic to characterize. Resonant orbits have been found with many different periods in that region (there are instances with 3,5,7,11 and many more multiples of the fundamental period), and there are many instances of multistability, as in $(S, R a)=(-0.9525,2181.2)$, where the LPNS orbit coexists with a period-18 resonance. The many resonance regions found, possibly due to the extreme difference between the fast and the slow frequency, obscure thus this transition to the point where we do not find one scenario but many. Furthermore, it has to be noted that the closer we are to the transition region the transients, which might be chaotic, become longer and longer, hence making more and 
more difficult to identify unequivocally the nature of the attractor.

For higher values of $R a$, as $S$ is increased, the nature of the disappearance of the attractor also changes. In a similarly obscure scenario, in the region in parameter space marked with $A$, the $B S C$ changes to a homoclinic torus bifurcation at the line marked with $h$ in Fig. 9. There are some evidences for the identification of the homoclinic torus bifurcation. On the one hand, the Poincare section performed on the temporal evolution shows that the solution is quasiperiodic, for values of $S$ high enough (to the right of the thick broken line in Fig. 9). On the other hand we measure a logarithmic divergence law for the slow period of the quasiperiodic solution when approaching the bifurcation. Again, it is not clear the nature of the change from BSC to homoclinic torus bifurcation, as very close to $A$ resonant zones again tend to accumulate, and longer and longer time series are needed to characterize the behavior of the system, a problem aggravated by the fact that the long period is divergent.

For values of $S$ high enough, the bifurcation scenario becomes much more clear, as the regions with periodic or quasiperiodic solutions are unambiguously identified. The $h$ bifurcation line turns towards higher values of $R a$, and there are no more global bifurcations present. For $S>-0.92$, if $R a$ is increased, the quasiperiodic solution disappears in a low-frequency backwards NS bifurcation, $N S_{2}$, where a short-period nonsymmetric orbit bifurcates. This orbit in turn disappears for high enough $R a$ in a saddle node of periodic orbits $(S N P O)$, and thus the only remaining solution is the symmetric orbit that had become stable in the pitchfork of periodic orbits $(P P O)$.

If we continue the three bifurcation lines $h, N S_{2}$ and $S N P O$ towards higher values of $R a$ and more negative values of $S$, using time evolution, we find that the long period of $N S_{2}$ becomes longer and longer as the three lines converge to a point around $(S, R a)=(-0.975,2474.73)$.

Given the nature of the bifurcation lines that converge at that point, we identify it as a codimension-two bifurcation, a Takens-Bogdanov point of periodic orbits (TBPO). Beyond this point, there are no more nonsymmetric solutions that are stable, and the only stable solution remaining is the symmetric periodic short-period orbit that becomes stable at PPO.

\subsection{High Ra. HH Point. Interaction of $P$ and $H_{\text {sym }}$. Codimension-three Dynamics.}

As $S$ is further increased, the lines corresponding to the two NS bifurcations introduced in the previous section, $N S_{1}$ and $N S_{2}$, converge in a 


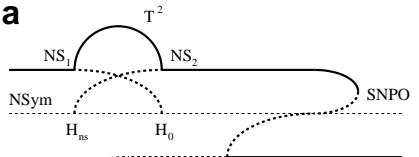

b

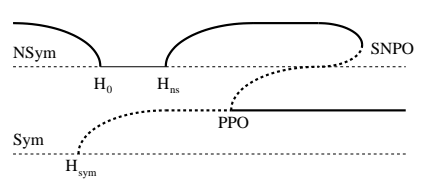

Figure 10: Enlargement of the dotted rectangle in Fig. 9. Dashed lines correspond to bifurcations between unstable states, found by continuation. In the insets a) to d) are represented diagrams of the solutions present in the corresponding constant- $S$ cuts. An enlargement of the small dotted rectangle near $S=-0.845$ between cuts c) and d) is shown in Fig. 11a

Hopf-Hopf bifurcation, $H H$. In Fig. 10 we show an enlargement of Fig. 9, with some bifurcation diagrams on the side to help discuss the dynamics.

First, in Fig. 10a we show a diagram of a representative cut of the dynamics before the $H H$ point for $S \approx-0.9$. We show the two unstable steady solutions as two parallel dashed lines, corresponding to the symmetric and the nonsymmetric branch of developed solutions shown in Fig. 2 for $S=-1$. At $H_{\text {sym }}$ a symmetric unstable orbit appears, which becomes stable at PPO, where an unstable nonsymmetric orbit appears.

Also, as it has been described in the previous section, the LPNS becomes unstable in a forward NS bifurcation $\left(N S_{1}\right)$ and then the resulting torus disappears in a reverse NS $\left(N S_{2}\right)$, where a short-period nonsymmetric orbit bifurcates. This orbit loses stability at $S N P O$, finally connecting with the $P P O$ point. The LPNS orbit, unstable for $R a>R a_{N S_{1}}$, disappears in a 
backward Hopf bifurcation $\left(H_{0}\right)$ of the nonsymmetric solution, which is the same through which it is created in the low- $R a$ regime for $S>S_{T B}$, see Section 3.2. The unstable short-period nonsymmetric orbit appears in the Hopf bifurcation $H_{n s}$ of the nonsymmetric steady solution before becoming stable at $N S_{2}$. This is the same bifurcation that was introduced in Section 3.1.

As we have mentioned before, $N S_{1}$ and $N S_{2}$ meet at $H H$. To fully characterize the point $H H$ we should locate the relative position of $H_{n s}$, $H_{0}, N S_{1}$ and $N S_{2}$. From the angle at which these bifurcation lines meet at $H H$ we conjecture that the sequence, in ascending $R a$, is $H_{n s^{-}} N S_{1^{-}}-S_{2^{-}}$ $H_{0}$, which corresponds to the (time-reversed) case $I a$ of the classification of Guckenheimer and Holmes [34].

In Fig. 10b we show the bifurcation diagram right after the $H H$ point. Now, the non-symmetric steady solution is stable again between $H_{0}$ and $H_{n s}$, which have inverted their order, and of course the torus has disappeared.

If we keep increasing $S$ we observe first that $P P O$ and $H_{n s}$ cross each other, but this is not a codimension-two bifurcation as they are bifurcations of different solutions. For $S=-0.84762, H_{n s}$ and $S N P O$ meet at a Degenerate Hopf / Bautin codimension-two point, $D H$, near $R a=2146.09$. At this point $H_{n s}$ changes, ceasing to be a supercritical Hopf to become subcritical. This is illustrated in Fig. 10c, where we show how the non-symmetric steady solution now does not become an orbit for $R a>R a_{H_{n s}}$ and hence beyond this point the only stable solution remaining is the symmetric periodic orbit, which becomes stable at the $P P O$ point. Also for this value of $S$, we find again the $P$ bifurcation that was present for low $R a$, and there is where the nonsymmetric steady solution branch ceases to exist as $R a$ is increased.

With a slight increase of $S$, the bifurcation landscape changes dramatically. As it is portrayed in Fig. 10d, now $H_{\text {sym }}$ and $P$ have exchanged their order, and the symmetric steady solution is stable again in the region between $P$ and $H_{\text {sym }}$. $P$ has changed from subcritical to supercritical, with an additional saddle node, and $H_{n s}$ and $P P O$ have disappeared altogether. In that small interval of $S$, many changes have taken place, obviously related with the pitchfork-Hopf $\left(P-H_{\text {sym }}\right)$ interaction.

In Fig. 11a we portray an enlargement of that small region, enclosed by a dotted rectangle near $S=-0.845$ in Fig. 10 .

As we see in the figure, as $S$ is increased, $P$ changes from a subcritical to a supercritical bifurcation in a degenerate pitchfork codimension-two bifurcation $(D P 1)$ at $S=-0.845084$ similar to the previously described $D P 2$ (see Section 3.3). At this point, the previously described $S N$ branch appears. 
a

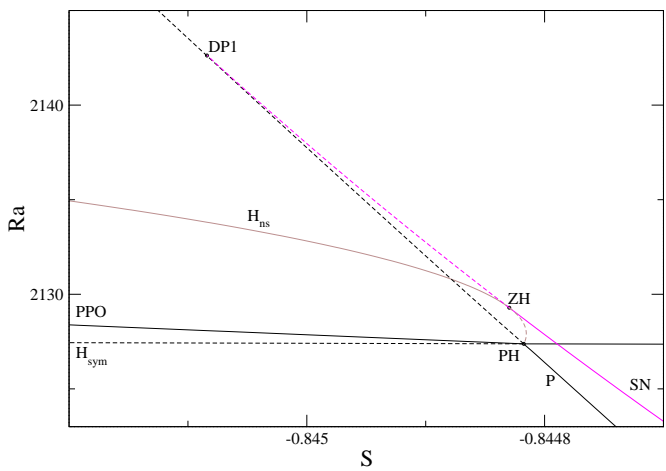

b

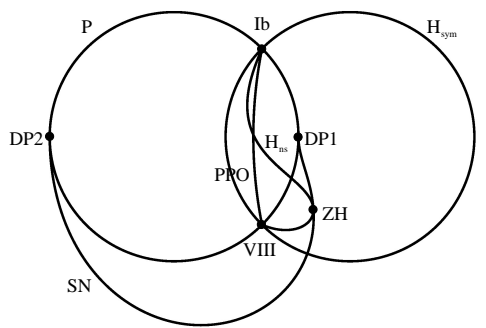

Figure 11: a) Enlargement small dotted rectangle from Fig. 10. Dashed lines correspond to bifurcations between unstable states, found by continuation. b) Diagram of the DHPiv case of the degenerate pitchfork-Hopf interaction, computed through a stereographic projection (see [35] and Appendix A).

This branch has a tangency point with the $H_{n s}$ branch at the $Z H$ point at $S=-0.844829$.

The $Z H$ point is a zero-Hopf (also fold-Hopf) codimension-two point. From the relative stability of the different solutions surrounding it, we characterize it as case $I V a / I V b$ of [34].

The $H_{n s}, P, H_{s y m}$ and $P P O$ branches meet at a pitchfork-Hopf point at $(S, R a)=(-0.844817,2127.38)(P H)$. The relative stability of the solutions (the symmetric steady, the nonsymmetric steady, the symmetric orbit, the non symmetric orbit) surrounding the $P H$ point lead us to identify it unambiguously with case VIII in the unfolding of the Hopf-Hopf bifurcation in [34]. Note that we can do that when it is not critical the fact that the normal form is four-dimensional, e.g. in the absence of resonances.

After the $P H$ point we recover the scenario depicted in Fig. 10d, but the fact that there are so many codimension-two bifurcations so close to each other has led us to conjecture that this scenario is related with the degeneracy of a codimension-three point, a higher codimension point where codimension-two bifurcations converge.

We have found a codimension-three candidate in a Degenerate pitchforkHopf $(D P H)$ point, and we identify it with case DHPiv from Algaba et al. [35] (in fact, they study a degenerate Hopf - pitchfork interaction, see Appendix A for the details of the correspondence). In Fig. 11b we show a diagram of the unfolding of this codimension-three point. Since it is a threedimensional parameter space, we are portraying the stereographic projection 
of the surface of a sphere surrounding the singular point. Also, similarly as in our bifurcation diagram, the lines identified as $H_{n s}, P P O$ and $P$ cross at noncodimension-two points. Note the similarity with our bifurcation diagram, as it also incorporates $D P 2$.

This association of codimension-two bifurcations acts as an organizing center for parameter space, and explains the transition from symmetric to nonsymmetric solutions as $S$ is decreased. In that respect we note that beyond $H_{n s}$ and $S N$ there are no more nonsymmetric solutions for higher $S$ and $R a$, in the studied parameter range, which helps make the connection with the $S=0$ case[1], where no such solutions were found. As we have seen in Section 3.3, in a different $R a$ interval, the last nonsymmetric solution is an isola associated with a turn in the $S N$ branch at about $S=-0.758955$.

Besides of the transition from symmetric to nonsymmetric solutions, the codimension-two bifurcations surrounding $P H$ help explain the different ways in which the fast frequency appears. In [2] it was noted how $H_{\text {sym }}$ and $H_{n s}$ have a very similar associated frequency at $S=-1$. The present study provides a connection between both of them, and also with PPO. Note that the stability boundary for the symmetric orbit at $S=0$ was $H_{\text {sym }}$ at $R a=$ 1991 [1], whereas for $S=-1$ it became stable at $P P O$, for $R a=2253.67$. The point $P H$ provides a transition between the two stability boundaries.

\section{Conclusions}

We have performed a comprehensive study of the dynamics of binarymixture convection on a laterally heated cavity, across a very large range of parameters, and we have connected the results reported for $S=0$ [1] with those for $S=-1[2,3]$, providing an interpretation in terms of dynamical systems. To this end we have studied both steady and time-dependent solutions (finding periodic, quasiperiodic and chaotic behaviors) by both time evolution and continuation of steady solutions, as discussed in Section 2.

We have calculated and described most of the codimension-two bifurcations of steady solutions found in that parameter range, and we have provided an interpretation in terms of higher codimension bifurcations in some cases, such as the DRS [24] or the DPH [35] bifurcations. Let us emphasize that as far as we know these later bifurcations had previously been found only in small-dimensionality ODE models, whereas in this work we have dealt with a space-dependent fluid system, as described by the PDE system given by Eqs. 
(1-4), which have been integrated directly. Comparable complex scenarios have been found in Taylor-Couette flows [36, 37, 38].

Also, we ascertain that the identification of higher codimension phenomena provide robustness. Small variations of the other parameters might give different unfoldings, but cannot change the nature of the singularity. Furthermore, the practical approach of conjecturing higher codimension phenomena helps focus the attention on small parts of parameter space, see e.g. Fig. 11. Without a prior idea of the codimension-two points to expect, the analysis and the continuation of these bifurcation lines would be very hard, given the very different scales present in parameter space. We note here a recent work discussing codimension-three bifurcations by Tuckerman [39].

Physically, the symmetric periodic solution that is born at $H_{\text {sym }}$ is only weakly dependent on the solute field, its development is related with the strength of the flow field, which in turn depends on the global thermal gradient. As $R a$ is increased it is the only remaining stable solution. Also, it is noteworthy that all the nonsymmetric solutions appear when $S$ is negative enough, relating symmetry with the ratio of buoyancies or with the Soret effect.

An important point that has to be addressed regarding these results is whether the bidimensionality of the system hinders the connection with experimental results. This question cannot be fully answered, as we know of no experimental realizations or three-dimensional simulations of the exact system of study, but we can extrapolate from what is known experimentally for the pure fluid and what has been found in prior numerical results. There are not many experiments of low-Prandtl number lateral convection. The fluids are often opaque, which makes it very difficult to measure their properties. Perhaps the most common material is gallium [40,41], studied to characterize the secondary instabilities in rectangular boxes. Experiments show a basic quasi 2D flow which becomes unstable in a Hopf bifurcation [40]. Both 3D and 2D simulations agree that the secondary instabilities are oscillating [40, 42], with a threshold depending strongly on the aspect ratio, but experiments show a supercritical Hopf bifurcation incompatible with numerical results, which is attributed to non-Boussinesq effects [40]. Hence, while simulations provide a good threshold for the secondary instability, they do not characterize properly the system, regardless of the dimensionality. Recent works $[43,44]$ have also characterized the secondary instabilities of low-Prandtl number fluids in a laterally heated cylinder. In particular, in Ref. [44] the 3D case is compared with the 2-dimensional problem for the 
same aspect ratio and Prandtl number [1], finding comparable results for the first bifurcation.

There exist some related numerical works that have been devoted to study the importance of the third dimension. For pure fluids and lateral convection, it has been studied the effect of three-dimensional disturbances on a $2 \mathrm{D}$ flow [30]. It was found, through a linear stability analysis, that the results depended critically of the Prandtl number. For higher Prandtl numbers the secondary instability remains 2D, but for smaller Prandtl number the 3D destabilization mechanism becomes more important, depending nevertheless on the aspect ratio. For the double-diffusive problem, in Ref. [18] the third dimension brings an extra branch of solutions, without changing the other branches except for their relative stability. In an intermediate range of $R a$, the problem reduces to the well-known two-dimensional problem $[45,14,15$, 17] when the vertical sides of the box parallel to the thermal gradient are far enough, so that even if they act as sources of vorticity this will be swamped by the two-dimensional density-driven flow when the box is long enough [18].

In conclusion, we expect that in a realistic setting, a basic steady quasi-2D flow followed by an oscillating instability for high enough Rayleigh number will develop, in correspondence with the pure fluid and the double-diffusive case. The nature of this oscillation will change when modifying the $3 \mathrm{D}$ shape of the box or when some physical aspects of the problem are included in the model, as it occurs in the previously cited experiments and numerics[40, 42]. Regarding the unstable and stable steady solutions that we find, we expect, as in the numerical examples for the double diffusive problem, that their stability will change when considering $3 \mathrm{D}$ aspects of the realistic problem, but the branches of the equivalent 2D solutions are likely to be present [30, 18], at least in rectangular boxes in which the presence of transverse walls has small influence. Lastly, little can be said about whether the periodic solutions will survive in a three-dimensional setting, but there is nothing that forbids them from having close analogues for limiting values of the aspect ratio.

Finally, despite the detail and the large range of parameters studied, this study still has some limitations, such as the full characterization of points $A$ (interaction $B S C$ - homoclinic torus) and $B$ (interaction flip - NeimarkSacker) in the bifurcation diagram (see Fig. 9). While the unfolding of the former is mostly unknown, there has been some theoretical work on the latter $[46,47,48]$, highlighting its complexity. This particular interaction has also been observed in molten gallium convection experiments [49]. As we have discussed, the huge difference between the two frequencies, long transients, 
divergent periods, multistability and the abundance of resonant solutions obscure the interpretation of $A$ and $B$. Hence, the characterization of these two points remains as future work.

\section{Acknowledgements}

E.M. acknowledges support from the Balsells Foundation and from the NSF Division of Mathematical Sciences through grant DMS-1217303. I.M. acknowledges support from DGICYT/FEDER under Grant FIS2009-08821 and from DGICYT under Grant FIS2013-40880-P. E.M. and L.R.-P. acknowledge support from DGICYT/FEDER under Grant FIS2012-37655-C02-01.

\section{Appendix A. Unfolding of the codimension-three singularity}

In this appendix we detail the correspondence between the DHPiv case from Algaba et al. [35], and the codimension-three bifurcation which, as we conjecture, gives an explanation of the association of many of the codimensiontwo bifurcations found in the intermediate $R a$ range.

While in [35] the case discussed is the interaction of a degenerate Hopf bifurcation with a pitchfork, their analysis is also valid for a degenerate pitchfork-Hopf interaction, in the same way as it is valid, to a certain extent, for nonlinear degenerate Hopf-Hopf interactions. It is trivial to adapt their results to our case, but we nevertheless do it, since the case of interest, DHPiv, is not discussed in detail in [35].

We start by writing down an unfolding of the singularity. The variable $z$ corresponds to the symmetry breaking and $\rho$ is a radial variable, corresponding to the radius of the periodic solution. The angular variable has been omitted, as the dynamics of $z$ and $\rho$ is decoupled from it (i.e. there is rotational symmetry).

$$
\begin{aligned}
& \dot{z}=z\left(\mu_{1}+\mu_{3} z^{2}+\rho^{2}+c z^{4}\right) \\
& \dot{\rho}=\rho\left(\mu_{2}+d z^{2}+a \rho^{2}\right)
\end{aligned}
$$

where $a \neq 0,1,2$ and $c= \pm 1, d= \pm 1$. Observe that this is simply Eq. (8) from reference [35], with $z$ and $\rho$ exchanged.

Taking different values of $a, b, c$ we obtain the different cases of the unfolding, all discussed in [35]. In order to identify which is the case that corresponds to ours we proceed in an indirect fashion. First, we identify the 
type of the codimension-two bifurcations that correspond to our results, and when that can only correspond to a possible case of the unfolding, we identify it.

In our case, we identified the $P H$ point in Fig. 11a as the case VIII in the unfolding of the Hopf-Hopf bifurcation, following Guckenheimer and Holmes [34]. Furthermore, we identify the $Z H$ point with the case $I V a / I V b$ for the Fold-Hopf bifurcation, following again the nomenclature of [34].

The previous identification of the $P H$ and $Z H$ bifurcations lead to an unambiguous identification among the degenerate pitchfork-Hopf bifurcations studied by Algaba et al. [35, 50, 51]. The case DHPiv from [35] is the only that corresponds to the explored bifurcation diagram, incorporating both codimension-two bifurcations in the specified case. Note that we do not prove that a projection of the global boundary value problem has this particular degeneracy, but we use this as a conjecture in order to further investigate parameter space. In our case, this led to the finding of the degenerate pitchfork bifurcations $D P 1$ and $D P 2$, that correspond directly with the ones found on Fig. 11b.

Figure 11b has been computed by finding the bifurcations of the steady solutions of Eqs. (A.1-A.2) in the case DH Piv $(a>0, c=+1, d=+1)$. In the three-dimensional parameter space spanned by $\left(\mu_{1}, \mu_{2}, \mu_{3}\right)$ we consider only the sphere given by $\mu_{1}^{2}+\mu_{2}^{2}+\mu_{3}^{2}=k^{2}$. We find the points (lines) where the codimension-two (one) bifurcations intersect the surface. In Fig. 11b we plot a stereographic projection of this sphere.

\section{References}

[1] I. Mercader, O. Batiste, L. Ramirez-Piscina, X. Ruiz, S. Rudiger, J. Casademunt, Bifurcations and chaos in single-roll natural convection with low Prandtl number, Phys. Fluids 17 (2005) 104108.

[2] E. Meca, I. Mercader, O. Batiste, L. Ramirez-Piscina, Blue sky catastrophe in double-diffusive convection, Phys. Rev. Lett. 92 (2004) 234501.

[3] E. Meca, I. Mercader, O. Batiste, L. Ramirez-Piscina, Complex dynamics in double-diffusive convection, Theoretical Computational Fluid Dynamics 18 (2004) 231-238.

[4] M. C. Cross, P. C. Hohenberg, Pattern-formation outside of equilibrium, Rev. Modern Phys. 65 (1993) 851-1112. 
[5] M. E. Stern, The salt-fountain and thermohaline convection, Tellus 12 (1960) 172-175.

[6] J. S. Turner, Double-diffusive phenomena, Ann. Rev. Fluid Mechanics 6 (1974) 37-56.

[7] S. M. Pimputkar, S. Ostrach, Convective effects in crystals grown from melt, J. Crystal Growth 55 (1981) 614-646.

[8] J. S. Turner, Multicomponent convection, Ann. Rev. Fluid Mechanics 17 (1985) 11-44.

[9] D. T. J. Hurle, E. Jakeman, Soret-driven thermosolutal convection, J. Fluid Mechanics 47 (1971) 667-687.

[10] E. Knobloch, Convection in binary fluids, Phys. Fluids 23 (1980) 19181920.

[11] M. E. Stern, Lateral mixing of water masses, Deep-sea Research 14 (1967) 747-753.

[12] S. A. Thorpe, P. K. Hutt, R. Soulsby, Effect of horizontal gradients on thermohaline convection, J. Fluid Mechanics 38 (1969) 375-400.

[13] D. Gobin, R. Bennacer, Double diffusion in a vertical fluid layer: Onset of the convective regime, Phys. Fluids 6 (1994) 59-67.

[14] K. Ghorayeb, A. Mojtabi, Double diffusive convection in a vertical rectangular cavity, Phys. Fluids 9 (1997) 2339-2348.

[15] S. H. Xin, P. Le Quéré, L. S. Tuckerman, Bifurcation analysis of doublediffusive convection with opposing horizontal thermal and solutal gradients, Phys. Fluids 10 (1998) 850-858.

[16] A. Bergeon, K. Ghorayeb, A. Mojtabi, Double diffusive instability in an inclined cavity, Phys. Fluids 11 (1999) 549-559.

[17] G. Bardan, A. Bergeon, E. Knobloch, A. Mojtabi, Nonlinear doubly diffusive convection in vertical enclosures, Physica D-Nonlinear Phenomena 138 (2000) 91-113. 
[18] A. Bergeon, E. Knobloch, Natural doubly diffusive convection in threedimensional enclosures, Phys. Fluids 14 (2002) 3233-3250.

[19] A. Bergeon, E. Knobloch, Spatially localized states in natural doubly diffusive convection, Phys. Fluids 20 (2008) 034102.

[20] A. Bergeon, E. Knobloch, Periodic and localized states in natural doubly diffusive convection, Physica D 237 (2008) 1139-1150.

[21] I. Mercader, O. Batiste, X. Ruiz, Quasi-periodicity and chaos in a differentially heated cavity, Theoretical Computational Fluid Dynamics 18 (2004) 221-229.

[22] M. T. Borisuk, J. J. Tyson, Bifurcation analysis of a model of mitotic control in frog eggs, J. Theoretical Biol. 195 (1998) 69-85.

[23] C. Mayol, R. Toral, C. Mirasso, M. Natiello, Class a lasers with injected signal: Bifurcation set and Lyapunov potential function, Phys. Rev. A 66 (2002) $013808(1-12)$.

[24] F. Dumortier, R. Roussarie, J. Sotomayor, Generic 3-parameter families of planar vector-fields, unfoldings of saddle, focus and ellipticsingularities with nilpotent linear parts, Lecture Notes In Mathematics 1480 (1991) R5-164.

[25] Y. A. Kuznetsov, Elements of Applied Bifurcation Theory, Springer, 1998.

[26] S. Hugues, A. Randriamampianina, An improved projection scheme applied to pseudospectral methods for the incompressible Navier-Stokes equations, Int. J. For Numerical Methods In Fluids 28 (1998) 501-521.

[27] C. K. Mamun, L. S. Tuckerman, Asymmetry and Hopf bifurcation in spherical Couette flow, Phys. Fluids 7 (1995) 80-91.

[28] A. Bergeon, D. Henry, H. Benhadid, L. S. Tuckerman, Marangoni convection in binary mixtures with Soret effect, J. Fluid Mechanics 375 (1998) 143-177.

[29] E. Chenier, C. Delcarte, G. Labrosse, Stability of the axisymmetric buoyant-capillary flows in a laterally heated liquid bridge, Physics of Fluids 11 (1999) 527-541. 
[30] S. H. Xin, P. Le Quéré, Linear stability analyses of natural convection flows in a differentially heated square cavity with conducting horizontal walls, Phys. Fluids 13 (2001) 2529-2542.

[31] V. Frayssé, L. Giraud, S. Gratton, J. Langou, A set of GMRES routines for real and complex arithmetics on high performance computers, Technical Report TR/PA/03/3, CERFACS, 2003. Public domain software available on www.cerfacs/algor/Softs.

[32] D. H. Kim, P. M. Adornato, R. A. Brown, Effect of vertical magneticfield on convection and segregation in vertical Bridgman crystal-growth, J. Crystal Growth 89 (1988) 339-356.

[33] P. M. Adornato, R. A. Brown, Convection and segregation in directional solidification of dilute and non-dilute binary alloys: Effects of ampoule and furnace design, J. Crystal Growth 80 (1987) 155-190.

[34] J. Guckenheimer, P. Holmes, Nonlinear Oscillations, Dynamical Systems and Bifurcations of Vector Fields, Springer, 1983.

[35] A. Algaba, E. Freire, E. Gamero, A. J. Rodriguez-Luis, On a codimension-three unfolding of the interaction of degenerate Hopf and pitchfork bifurcations, Int. J. Bifurcation Chaos 9 (1999) 1333-1362.

[36] J. Lopez, F. Marques, J. Shen, Complex dynamics in a short annular container with rotating bottom and inner cylinder, J. Fluid Mechanics 501 (2004) 327-354.

[37] J. Abshagen, J. Lopez, F. Marques, G. Pfister, Bursting dynamics due to a homoclinic cascade in taylor-couette flow, J. Fluid Mechanics 613 (2008) 357-384.

[38] F. Marques, A. Meseguer, J. Lopez, J. Pacheco, J. Lopez, Bifurcations with imperfect $\mathrm{SO}(2)$ symmetry and pinning of rotating waves, Proc. Royal Soc. London Series A - Mathematical Phys. Sciences 469 (2013) 20120348.

[39] L. S. Tuckerman, Amplitudes from eigenvalues, Fluid Dynamics Research 44 (2012) 031202. 
[40] B. Hof, A. Juel, L. Zhao, D. Henry, H. B. Hadid, T. Mullin, On the onset of oscillatory convection in molten gallium, J. Fluid Mechanics 515 (2004) 391-413.

[41] A. Juel, T. Mullin, H. B. Hadid, D. Henry, Three-dimensional free convection in molten gallium, J. Fluid Mechanics 436 (2001) 267-281.

[42] D. Henry, H. BenHadid, Multiple flow transitions in a box heated from the side in low-Prandtl-number fluids, Phys. Rev. E 76 (2007) 016314.

[43] S. Vaux, H. BenHadid, D. Henry, Study of the hydrodynamic instabilities in a differentially heated horizontal cylinder corresponding to a Bridgman growth configuration, J. Crystal Growth 290 (2006) 674-682.

[44] I. Mercader, O. Sánchez, O. Batiste, Secondary flows in a laterally heated horizontal cylinder, Physics of Fluids 26 (2014) 014104.

[45] D. Gobin, R. Bennacer, Cooperating thermosolutal convection in enclosures. II Heat transfer and flow structure, Int. J. Heat Mass Transfer 39 (1996) 2683-2697.

[46] Y. A. Kuznetsov, H. G. E. Meijer, Remarks on interacting NeimarkSacker bifurcations, J. Difference Equations Applications 12 (2006) 1009-1035.

[47] G. Mircea, D. Opris, Neimark-sacker and flip bifurcations in a discretetime dynamic system for Internet congestion, WSEAS Transactions On Mathematics 8 (2009) 63-72.

[48] S. Yao, New Bifurcation Critical Criterion of Flip-Neimark-Sacker Bifurcations for Two-Parameterized Family of n-Dimensional Discrete systems, Discrete Dynamics In Nature Soc. (2012) 264526.

[49] K. E. McKell, D. S. Broomhead, R. Jones, D. T. J. Hurle, Torus doubling in convecting molten gallium, Europhysics Lett. 12 (1990) 513-518.

[50] A. Algaba, E. Freire, E. Gamero, A. J. Rodriguez-Luis, A threeparameter study of a degenerate case of the Hopf-pitchfork bifurcation, Nonlinearity 12 (1999) 1177-1206. 
[51] A. Algaba, E. Freire, E. Gamero, A. J. Rodriguez-Luis, A tame degenerate Hopf-pitchfork bifurcation in a modified van der Pol-Duffing oscillator, Nonlinear Dynamics 22 (2000) 249-269. 